\title{
Influencia de las condiciones ambientales de la ciudad de Bogotá sobre el comportamiento mecánicos de una mezcla asfáltica
}

\section{Influence of the Bogotá environmental conditions on the mechanical behavior of an asphalt mixture}

\author{
Hugo Alexander Rondón Q uintana *1, Fredy Alberto Reyes Lizcano**
}

* Universidad Católica de Colombia. CO LO MBIA

** Pontificia Universidad Javeriana. CO LO M BIA

Fecha de recepción: 05/ 05/2009 Fecha de aceptación: 30/ 07/ 2009

Resumen PAG. 195 - 207

\begin{abstract}
El artículo presenta el cambio que experimenta el módulo resiliente, la deformación permanente y la resistencia última bajo carga monotónica de una mezcla asfáltica densa en caliente cuando es expuesta al ambiente de la ciudad de Bogotá D.C. (Colombia). Las mezclas fueron elaboradas y evaluadas empleando dos cementos asfálticos (CA) y un CA modificado (CAM) con polímero tipo etil vinil acetato: CA 80-100, CA 60-70 y CAM 20-40 respectivamente. Con el fin de observar su evolución en el tiempo, a la fecha los parámetros mecánicos de las mezclas han sido medidos cada tres meses aproximadamente durante 20 meses para el caso de las mezclas fabricadas con los CA 80-100 y CA 60-70, y 10 meses para aquellas que emplearon el CAM 20-40. De los resultados se concluye que el comportamiento que experimentan las mezclas depende del tipo de CA utilizado. Adicionalmente, la tendencia de las mezclas con los tres tipos de CA es experimentar un aumento en los valores de rigidez con el tiempo de exposición al ambiente debido principalmente a procesos de endurecimiento por envejecimiento de los ligantes asfálticos.
\end{abstract}

Palabras Clave: Módulo resiliente, deformación permanente, envejecimiento, cemento asfáltico, mezclas asfálticas

Abstract

The paper presents the evolution of the resilient modulus, permanent deformation and strength under monotonic loading of a dense hot mixture due to long term exposure to the environment in the city of Bogota D.C. (Colombia). The mixtures were made using two asphalt cements (AC) and modified asphalt cement (MAC) with ethil-vinil-acetate: AC 80-100, AC 60-70 and MAC 20-40 respectively. The mechanical properties have been evaluated every three months over a period of 20 months for the AC 80-100 and AC 60-70, and 10 months for MAC 20-40. The results show differences depending on the type of cement. All mixtures increase the modulus with time due to aging of the asphalt cement.

Keywords: Resilient modulus, permanent deformation, aging, asphalt cement, asphalt mixtures

\section{Introducción}

Los principales factores que afectan la durabilidad de las mezclas asfálticas, asumiendo que se encuentran bien construidas son: la edad de envejecimiento y el daño por humedad (Airey, 2003). Es decir, una mezcla asfáltica debe ser diseñada y construida no solo para que resista las cargas impuestas por el tránsito sino también la acción del medio ambiente. Cuantificar la influencia que tiene el ambiente sobre el comportamiento de este tipo de material no es una tarea fácil. La forma como se realiza actualmente es separando cada uno de los componentes que lo conforman (agua, temperatura y rayos ultra-violeta entre otros) y

\section{Introduction}

The main factors that affect durability of asphalt mixtures, assuming that it was properly made, are: aging and humidity damage (Airey, 2003). Then asphalt mixtures have to be designed and made not only to bear traffic loading but also environmental action. Quantifying the environmental influence over this material's behaviour is not an easy task. Nowadays, the way to quantify this considers separating every component (water, temperature, ultraviolet rays among others), and

\footnotetext{
${ }^{1}$ Autor de correspondencia / Corresponding author: E-mail: harondon@ucatolica.edu.co
} 
evaluar la influencia de cada uno de ellos (desde el punto de vista mecánico y químico) de manera separada sobre el ligante y las mezclas (Kemp y Predoehl, 1981; Welborn, 1984; Kim et al., 1987; Shiau et al., 1991; Bishara et al., 2000; Bocci y Cerni, 2000; Brown y Scholz, 2000; Khalid y Walsh, 2000; Khalid, 2002; Airey, 2003; Said, 2005; Shen et al., 2006). Adicionalmente por lo general estos estudios utilizan ensayos y equipos (p.e., hornos para ensayo de película delgada del tipo TFOT o rotatorios RTFOT, microondas) que no pueden reproducir totalmente la influencia que tiene cada uno de los componentes del ambiente sobre las propiedades mecánicas y reológicas de las mezclas y los ligantes asfálticos (Jemison et al., 1991; Choquet y Verhasselt, 1992; Kuppens et al., 1997; Verhasselt, 1997). Incluso la mayor parte de las investigaciones combinan estos ensayos y equipos para intentar evaluar la forma como cambian las propiedades de estos materiales cuando experimentan condiciones reales del ambiente (Jemison et al., 1991; Migliori y Corté, 1998; Montepara, 1999; M ontepara y Giuliani, 2000; Airey, 2003). En conclusión general se reporta que estas metodologías de ensayo tienen como limitación principal que son incapaces aún de reproducir las condiciones reales a las cuales están expuestas las mezclas asfálticas in situ.

Una forma de medir in situ la influencia que tiene el ambiente en el comportamiento de mezclas asfálticas es realizando tramos de prueba o pistas de prueba a escala. La limitación de este tipo de pruebas radica en que son muy costosas y en ellas no se puede medir de manera directa y por separado, la influencia que tienen las cargas vehiculares y el ambiente. Por lo anterior, el presente trabajo de investigación tiene como objetivo principal realizar una serie de ensayos en los cuales la mezcla asfáltica es sometida a solicitaciones reales de temperatura, precipitación, rayos ultravioletas, aire, etc. durante cinco años, para evaluar la influencia de estas condiciones en su comportamiento mecánico. En este artículo se presenta la evolución del módulo resiliente, la resistencia a la deformación permanente y los parámetros de estabilidad y rigidez Marshall de mezclas asfálticas densas en caliente tipo MDC-2 (de acuerdo a INVIAS (2007) cuando fueron expuestas al ambiente de la ciudad de Bogotá D.C. Se optó por utilizar este tipo de mezcla debido a que son las más utilizadas en Colombia para conformar capas de rodadura (las cuales son las que se encuentran sometidas de manera directa a las condiciones del ambiente). De la misma forma, las condiciones climáticas de Bogotá D.C. evaluates every element's influence (from a chemical and mechanic point of view) separating binder and mixtures (Kemp y Predoehl, 1981; Welborn, 1984; Kim et al., 1987; Shiau et al., 1991; Bishara et al., 2000; Bocci y Cerni, 2000; Brown y Scholz, 2000; Khalid y Walsh, 2000; Khalid, 2002; Airey, 2003; Said, 2005; Shen et al., 2006). Additionally, these researches usually use tests and equipments (e.g. thin film oven tests TFO T or rolling thin-film oven tests RTFOT, microwaves) that are not able to fully reproduce the environmental influence of each component on the mechanic and rheological mixtures and asphalt binders (Jemison et al., 1991; Choquet y Verhasselt, 1992; Kuppens et al., 1997; Verhasselt, 1997). Most researches, even analyze these tests and equipments trying to evaluate the way how this material properties change at facing real environmental conditions. (Jemison et al., 1991; Migliori y Corté, 1998; Montepara, 1999; Montepara y Giuliani, 2000; Airey, 2003). The general conclusion shows that the main limitation of these methodologies is being unable to reproduce the actual conditions that asphalt mixture faces in situ.

One way to measure in situ the environment influence over asphalt mixtures behaviour is using test sample areas or scale test tracks. The limitation of these types of tests is that they are costly and that they do not allow to measure directly or separatly the influence of vehicle loadings and the environment. For this reason this research main objective is to make several tests to put asphalt mixture under real temperature requirements, precipitations, ultra violet rays, air, and others during five years, to evaluate the influence of these conditions over mechanical behaviour. This paper shows the evolution of the resilient modulus, resistance to permanent deformation, Marshall Stabilization and stiffness parameters of hot asphalt dense mixtures MDC-2 (according to INVIAS (2007) when this was exposed to Bogotá D.C environment. The choice was using this type of mixture that is the most commonly used in Colombia for pavement layers (which faces directly environmental conditions). Bogotá weather conditions were considered 
fueron escogidas debido principalmente a que la misma se encuentra en una zona donde se presentan las siguientes condiciones ambientales en un día determinado: clima predominantemente frío con temperaturas mínimas y máximas promedio de $5^{\circ} \mathrm{C}$ y $19^{\circ} \mathrm{C}$ respectivamente y presencia de lluvias periódicas en cualquier momento del día. Con el fin de entender con mayor precisión la influencia del ambiente en el comportamiento de mezclas asfálticas, en futuras investigaciones se analizarán otros climas diferentes.

En los primeros 20 meses del proyecto se han evaluado las mezclas fabricadas con cemento asfáltico (CA) tipo CA 60-70 y CA 80-100. De la misma forma se presenta la evaluación de los primeros 10 meses del proyecto para mezclas fabricadas con CA modificado (CAM) tipo CAM 20-40 (denominado como Mexphalte $A M)$, que utiliza como modificador un polímero denominado etil vinil acetato. La diferencia en los tiempos de evaluación radica en que inicialmente el estudio se proyectó para valorar los dos tipos de CA producidos en Colombia y nueve meses después se identificó la necesidad de valorar adicionalmente un CAM que presentara una consistencia similar al CA 60-70, con el fin de comparar los resultados que se habían obteniendo con este tipo de asfalto a la fecha, los cuales no tenían una tendencia y significado físico claramente definido.

\section{Materiales y metodología}

El agregado pétreo empleado para la elaboración de las mezclas asfálticas para el ensayo Marshall (llamadas briquetas) es procedente de la cantera "Subachoque" (Cundinamarca, Colombia). A estos materiales se le realizaron los siguientes ensayos, siguiendo las especificaciones del Instituto Nacional de Vías (INVIAS, 2007a): Análisis granulométrico de agregados gruesos y finos (INV. E - 213), peso específico y absorción de agregados finos (IN V. E - 222), peso específico y absorción de agregados gruesos (INV. E - 223), resistencia al desgaste de los agregados (tamaños menores de $3 / 4^{\prime \prime}$ ) por medio de la máquina de Los Ángeles (INV. E - 218), desgaste M icroDeval (INV. E-238), pérdida en ensayo de solidez (IN V. E-220), partículas fracturadas (IN V. E - 227), ensayo para medir el equivalente de arena (INV. E - 133) e índices de alargamiento y aplanamiento (INV. E-230). Los resultados de estos ensayos se presentan en la Tabla 1 y se observa que los valores cumplen con los requisitos mínimos de calidad exigidos por las especificaciones IN VIAS (2007) para fabricar mezclas tipo M DC-2 para capas de rodadura. as an option due that this city is placed in an area that shows the following environmental features in one determined day: weather predominantly cold with minimum and maximum average temperatures within $5^{\circ{ }^{\circ} \mathrm{C}}$ y $190^{\circ} \mathrm{C}$, respectively, and frequent rains at any moment along the day. In the future it is considered to analyse areas with different weather conditions to better understand the environment influence over asphalt mixtures.

During the first 20 months of this project the evaluation has been focussed on asphalt cement (AC) mixtures, type $A C$ 60-70 and AC 80-100. The evaluation for ten months of mixtures that contain modified $A C$ (ACM) type ACM 20-40 (called Mexphalte AM), which is used as a polymer modifier called ethil-vinil-acetate, is also included. This difference in time evaluation is because the initial objective was to calculate the two types of AC produced in Colombia, and then nine months later there arises the need to additionally calculate the ACM that presented a similar formation to AC 60-70, the idea was to compare the results already obtained with this type of asphalt, which lacked a clearly defined tendency or a physical defined meaning.

\section{Methodology and Materials}

The stone aggregate, originally from "Subachoque" quarry (Cundinamarca, Colombia), was used to built asphalt mixtures for Marshall Test (specimens). According to the Instituto $\mathrm{N}$ acional de Vias specifications (INVIAS, 2007a), this material was tested accoring: Fine and coarse aggregate sieve analysis (IN V. E - 213), specific weight and fine aggregate absorption (IN V. E - 222), specific weight and coarse aggregate absorption (IN V. E - 223), aggregate abrasion strength (size smaller than $3 / 4^{\prime \prime}$ ) using Los Angeles machine (INV. E - 218), MicroDeval abrasion (NV. E-238), sulfate soundness (INV. E220), fractured particles (INV. E - 227), test to measure clay equivalent (INV. E - 133), shape index test (INV. E230). Table 1 shows the results reached and we can see that values accomplish the minimum quality requirements that IN VIAS (2007) specification demands, to build dense MDC-2 mixtures for pavement layers. 
Tabla 1. Caracterización de los agregados Table 1. Aggregate Properties

\begin{tabular}{|l|c||}
\hline EN SAYO/TEST & VALO R/VALUE \\
\hline Peso específico/Specific Weight & 2.56 \\
\hline Equivalente de arena/Clay equivalent & $86 \%$ \\
\hline Caras fracturadas/Fractured faces (surfaces) & $92 \%$ \\
\hline Índice de alargamiento/Shape index test (large) & $10 \%$ \\
\hline Índice de aplanamiento/Shape index test (level) & $10 \%$ \\
\hline Ataque en Sulfato de sodio/Sulfate soundness & $12.4 \%$ \\
\hline M icrodeval (sin saturar)/Microdeval (without saturation) & $20.3 \%$ \\
\hline $\begin{array}{l}\text { Resistencia al desgaste máquina de los Ángeles/ } \\
\text { Los Angeles machine abrasion strength }\end{array}$ & $22.5 \%$ \\
\hline
\end{tabular}

A los cementos asfálticos no modificados se les realizaron los ensayos típicos que exige la especificación INVIAS (2007) para caracterizarlos como son: penetración, viscosidad absoluta, ductilidad, solubilidad en tricloroetileno, contenido de agua, punto de ablandamiento y ensayos al residuo luego del ensayo de película delgada en horno rotatorio (RTFOT por sus siglas en inglés). Al cemento asfáltico modificado adicionalmente se le realizó el ensayo de recuperación elástica por torsión. Los resultados de estos ensayos se presentan en las Tablas 2-4.

Luego de realizar los ensayos al agregado pétreo y a los cementos asfálticos se fabricaron cinco briquetas (compactadas a 75 golpes por cara) para cada porcentaje de asfalto entre 4.5 y $6.5 \%$ con el fin de realizar el diseño Marshall (IN V. E-748, IN VIAS, 2007a) para determinar el contenido óptimo de asfalto de las mezclas convencionales. Para cumplir con las especificaciones del INVIAS (2007) y fabricar mezclas asfálticas tipo M DC2 , se modificó la granulometría original de los agregados, tomando como referencia los valores promedios en porcentajes de la franja granulométrica que exige la especificación para la elaboración de las briquetas del ensayo Marshall. Luego de realizar el diseño Marshall se elaboraron las briquetas (180 por tipo de CA) de mezcla asfáltica tipo MDC-2 necesarias para exponerlas al ambiente de la ciudad de Bogotá D.C. Estas muestras fueron colocadas en el techo del edificio de laboratorios de la Facultad de Ingeniería de la U niversidad Católica de Colombia. Las muestras que recibieron de manera directa la influencia del ambiente fueron ensayadas cada tres meses durante los primeros 20 meses del proyecto para el caso de las mezclas fabricadas con los CA 80100 y CA 60-70, y 10 meses para aquellas que emplearon el CA modificado (CAM 20-40).
The non modified asphalt cements and the modified asphalt cement were tested according to IN VIAS (2007) specification requirements: penetration test, absolute viscosity test, ductility test, solubility in trichloroethylene test, water content test, softening point test and residual test after RTFOT. Additionally, modified asphalt cement was tested with twist elastic recovery. These test results are shown in Tables 2 through 4.

After testing stone aggregate and asphalt cement, five marshall specimens were made (compacted with 75 hits per face) for each asphalt percentage between 4.5 y $6.5 \%$, with the idea of doing Marshall design (INV. E-748, INVIAS, 2007a), in order to determine the optimum asphalt conventional mixture state. The original aggregate size distribution was modified to accomplish INVIAS (2007) specification to obtain a MDC-2 mixture based on the average values of the size range required for tha $M$ arshall Test. The elaboration of specimens came after the MDC-2 Marshall design, (180 per type AC), mixture suitable to be exposed at Bogotá city environment. These samples were placed in the roof of the Facultad de Ingeniería de la U niversidad Católica de Colombia. The specimens that received direct environmental influence were tested every three months, during the first 20 months at the beginning of this project, as the case of $A C 80$ 100 and $A C$ 60-70, and those using modified AC (ACM 20-40) during the 10 first months. 
Para evaluar las características dinámicas de las mezclas se realizaron ensayos de módulo resiliente y deformación permanente. El ensayo de módulo resiliente (INV. E-749, INVIAS, 2007) fue realizado a tres temperaturas $\left(10,20\right.$ y $\left.30^{\circ} \mathrm{C}\right)$ y frecuencias de carga diferentes $(2.5,5.0$ y $10.0 \mathrm{~Hz})$ utilizando un equipo $\mathrm{N}$ ottingham Asphalt Tester (NAT), y el de resistencia a la deformación permanente bajo carga repetida fue realizado bajo un esfuerzo de 100 kPa y a 3600 ciclos de carga siguiendo el procedimiento normalizado por el CEN (2000, EN 12697-22).
Resilience modulus and permanent deformation tests were performed to evaluate the mixture dynamic characteristics. The resilience modulus test, (INV. E-749, INVIAS, 2007), was made at three temperatures (10, 20 y $\left.30^{\circ} \mathrm{C}\right)$, with different loading frequencies $(2.5,5.0 \mathrm{y}$ $10.0 \mathrm{~Hz}$ ) using a Nottingham Asphalt Tester (NAT) equipment, and the resistance to permanent deformation was tested under loading cycling with a stress of $100 \mathrm{kPa}$ at 3600 cycles in compliance with the standard CEN (2000, EN 12697-22).

Tabla 2. Características generales del cemento asfáltico CA 80-100

Table 2. General Asphalt Cement Characteristics AC80-100

\begin{tabular}{|c|c|c|c|c|}
\hline Ensayo/Test & Método/M ethod & Unidad/U nit & CA $80-100 / A C 80-100$ & Resultado/Result \\
\hline \multicolumn{5}{|c|}{ Ensayos sobre el asfalto original/Test on the O riginal Asphalt } \\
\hline Penetración $\left(25^{\circ} \mathrm{C}, 100 \mathrm{~g}, 5 \mathrm{~s}\right) /$ Penetration $\left(25^{\circ} \mathrm{C}, 100 \mathrm{~g}, 5 \mathrm{~s}\right)$ & ASTM D-5 & $0.1 \mathrm{~mm}$ & $80-100$ & 85 \\
\hline Índice de penetración/Penetration rate & INV. E-724 & - & $-1 /+1$ & -0.5 \\
\hline Viscosidad absoluta $\left(60^{\circ} \mathrm{C}\right) /$ Absolute Viscosity $\left(60^{\circ} \mathrm{C}\right)$ & ASTM D-4402 & Poises & 1000 mín. & 1400 \\
\hline Ductilidad $\left(25^{\circ} \mathrm{C}, 5 \mathrm{~cm} / \mathrm{min}\right) /$ Ductility $\left(25^{\circ} \mathrm{C}, 5 \mathrm{~cm} / \mathrm{min}\right)$ & ASTM D-113 & $\mathrm{cm}$ & 100 mín. & $>105$ \\
\hline Solubilidad en Tricloroetileno/Solubility in trichloroethylene & ASTM D-2042 & $\%$ & 99 mín. & $>99$ \\
\hline Contenido de agua/Water content & ASTM D-95 & $\%$ & 0.2 máx. & $<0.2$ \\
\hline Punto de inflamación COC/COC Flash point & ASTM D-92 & ${ }^{\circ} \mathrm{C}$ & 232 mín. & 295 \\
\hline \multicolumn{5}{|c|}{ Ensayos sobre el residuo luego del RTFOT/Tests on the residual elements after RTFOT } \\
\hline Pérdida de masa/M ass lost & ASTM D-2872 & $\%$ & 1.0 máx. & 0.2 \\
\hline Penetración $\left(25^{\circ} \mathrm{C}, 100 \mathrm{~g}, 5 \mathrm{~s}\right) /$ Penetration $\left(25^{\circ} \mathrm{C}, 100 \mathrm{~g}, 5 \mathrm{~s}\right)$ & ASTM D-5 & $\%$ & 48 mín. & 65 \\
\hline
\end{tabular}

Tabla 3. Características generales del cemento asfáltico CA 60-70 Table 3. General characteristics of asphalt cement AC 60-70

\begin{tabular}{|c|c|c|c|c|}
\hline Ensayo/Test & M étodo/M ethod & Unidad/Unit & CA $80-100 / A C 60-70$ & Resultado/Result \\
\hline \multicolumn{5}{|c|}{ Ensayos sobre el asfalto original/Test on the O riginal Asphalt } \\
\hline Penetración $\left(25^{\circ} \mathrm{C}, 100 \mathrm{~g}, 5 \mathrm{~s}\right) /$ Penetration $\left(25^{\circ} \mathrm{C}, 100 \mathrm{~g}, 5 \mathrm{~s}\right)$ & ASTM D-5 & $0.1 \mathrm{~mm}$ & $60-70$ & 67 \\
\hline Índice de penetración/Penetration rate & INV. E-724 & - & $-1 /+1$ & -0.7 \\
\hline Viscosidad absoluta $\left(60^{\circ} \mathrm{C}\right) /$ Absolute Viscosity $\left(60^{\circ} \mathrm{C}\right)$ & ASTM D-4402 & Poises & 1500 mín. & 1750 \\
\hline Ductilidad $\left(25^{\circ} \mathrm{C}, 5 \mathrm{~cm} / \mathrm{min}\right) /$ Ductility $\left(25^{\circ} \mathrm{C}, 5 \mathrm{~cm} / \mathrm{min}\right)$ & ASTM D-113 & $\mathrm{cm}$ & 100 mín. & $>105$ \\
\hline Solubilidad en Tricloroetileno/Solubility in trichloroethylene & ASTM D-2042 & $\%$ & 99 mín. & $>99$ \\
\hline Contenido de agua/Water content & ASTM D-95 & $\%$ & 0.2 máx. & $<0.2$ \\
\hline Punto de inflamación COC/COC Flash point & ASTM D-92 & ${ }^{\circ} \mathrm{C}$ & 232 mín. & 275 \\
\hline \multicolumn{5}{|c|}{ Ensayos sobre el residuo luego del RTFOT/Tests on the residual elements after RTFOT } \\
\hline Pérdida de masa/M ass lost & ASTM D-2872 & $\%$ & 1.0 máx. & 0.4 \\
\hline Viscosidad absoluta $\left(60^{\circ} \mathrm{C}\right) /$ Absolute Viscosity $\left(60^{\circ} \mathrm{C}\right)$ & ASTM D-4402 & Poises & 10000 máx. & 5200 \\
\hline Índice de durabilidad/D urability rate & - & - & 3 máx. & $<3$ \\
\hline Penetración $\left(25^{\circ} \mathrm{C}, 100 \mathrm{~g}, 5 \mathrm{~s}\right) /$ Penetration $\left(25^{\circ} \mathrm{C}, 100 \mathrm{~g}, 5 \mathrm{~s}\right)$ & ASTM D-5 & $\%$ & 52 mín. & 70 \\
\hline
\end{tabular}

Tabla 4. Características generales del cemento asfáltico CAM 20-40 Table 4. General characteristics of asphalt cement M AC 20-40

\begin{tabular}{|c|c|c|c|c|}
\hline Ensayo/Test & Método/M ethod & Unidad/U nit & CA $20-40 /$ AC $20-40$ & Resultado/Result \\
\hline \multicolumn{5}{|c|}{ Ensayos sobre el asfalto original/Test on the O riginal Asphalt } \\
\hline Penetración $\left(25^{\circ} \mathrm{C}, 100 \mathrm{~g}, 5 \mathrm{~s}\right) /$ Penetration $\left(25^{\circ} \mathrm{C}, 100 \mathrm{~g}, 5 \mathrm{~s}\right)$ & ASTM D-5 & $0.1 \mathrm{~mm}$ & $20-40$ & 37 \\
\hline Punto de ablandamiento (R\&B)/Softening Point (R\&B) & ASTM D-36 & ${ }^{\circ} \mathrm{C}$ & 60 mín. & 74 \\
\hline Recuperación elástica por torsión a 25ㄷ/Twisting elastic recovery at $25^{\circ} \mathrm{C}$ & NLT-329 & $\%$ & 10 mín. & 35 \\
\hline Punto de inflamación COC/COC Flash point & ASTM D-92 & ${ }^{\circ} \mathrm{C}$ & 230 mín. & 300 \\
\hline \multicolumn{5}{|c|}{ Ensayos sobre el residuo luego del RTFOT/Tests on the residual elements after RTFOT } \\
\hline Pérdida de masa/M ass lost & ASTM D-2872 & $\%$ & 1.0 máx. & 0.2 \\
\hline Penetración $\left(25^{\circ} \mathrm{C}, 100 \mathrm{~g}, 5 \mathrm{~s}\right) /$ Penetration $\left(25^{\circ} \mathrm{C}, 100 \mathrm{~g}, 5 \mathrm{~s}\right)$ & ASTM D-5 & $\%$ & 70 mín. & 76 \\
\hline
\end{tabular}


Durante la realización de los ensayos (mayo de 2007 a enero de 2009), las temperaturas medias máximas y mínimas de la ciudad de Bogotá D.C. estuvieron entre 18 y $19^{\circ} \mathrm{C}$, y entre 5 y $7^{\circ} \mathrm{C}$ respectivamente. La precipitación media varío entre 33 y $112 \mathrm{~mm} / \mathrm{mes}$, presentándose los mayores valores en los meses de septiembre, octubre y noviembre en los dos años.

\section{Resultados y análisis}

\subsection{Ensayo Marshall}

Los cálcul os obtenidos del ensayo Marshall para las briquetas elaboradas con asfalto convencional CA 80 100, CA 60-70 y CAM 20-40 están registrados en las Tablas 5-7 respectivamente.

Los porcentaje óptimos de cemento asfáltico de acuerdo con los datos de las tablas 5-7 son de 5.3\%, $5.6 \%$ y $5.6 \%$ para el caso de mezclas fabricadas con CA 80-100, CA 60-70 y CAM 20-40 respectivamente. En estos porcentajes se están cumpliendo, los requisitos mínimos exigidos por la especificación IN VIAS (2007) para MDC-2 y tránsitos tipo NT1 y/o NT2.
The mean, maximum and minimum Bogotá's temperature were between 18 and $19^{\circ} \mathrm{C}$, and 5-7을 respectively during these tests ( May 2007 to January 2009). The variation of mean precipitation was within 33 y $112 \mathrm{~mm} /$ monthly, the main values were in September, $O$ ctober and November these two years.

\section{Analysis and Results}

\subsection{Marshall Test}

The calculations obtained with Marshall test for specimens made with traditional asphalt AC 80-100, AC 60-70 and ACM 20-40 are registered in Tables 5 through 7 , respectively.

The optimum percentages of asphalt cement, according to the records in Tables 5 through 7, are 5.3\%, $5.6 \%$ and $5.6 \%$ for mixtures elaborated with AC $80-100$, AC 60-70 and ACM 20-40 respectively. These percentages accomplish the minimum requirements demanded by INVIAS (2007) specification for dense MDC-2 and transit type NT1 and/or NT2.

Tabla 5. Resumen del ensayo Marshall para mezcla asfáltica MDC-2 con CA 80-100 Table 5. Marshall Test summary to asphalt mixture MDC-2 with AC 80-100

\begin{tabular}{||c|c|c|c|c|c|c||}
\hline $\begin{array}{c}\text { CA } \\
\text { AC } \\
{[\%]}\end{array}$ & $\begin{array}{c}\text { Peso unitario } \\
\text { Unit weight } \\
{\left[\mathrm{g} / \mathrm{cm}^{3}\right]}\end{array}$ & $\begin{array}{c}\text { Estabilidad } \\
\text { Stability } \\
(\mathrm{E})[\mathrm{kg}]\end{array}$ & $\begin{array}{c}\text { Vacíos en la mezcla } \\
\text { Vacuum M ixture } \\
{[\%]}\end{array}$ & $\begin{array}{c}\text { Vacíos en agregados } \\
\text { Vacuum on aggregate } \\
{[\%]}\end{array}$ & $\begin{array}{c}\text { Flujo/Fluid } \\
(\mathrm{F}) \\
{[\mathrm{mm}]}\end{array}$ & $\begin{array}{c}\text { Relación } \\
\text { Relation } \\
\text { E/F }[\mathrm{kg} / \mathrm{mm}]\end{array}$ \\
\hline 4.5 & 2.21 & 710 & 7.63 & 17.52 & 3.33 & 213.09 \\
\hline 5.0 & 2.24 & 835 & 5.76 & 16.89 & 3.45 & 242.14 \\
\hline 5.5 & 2.24 & 853 & 5.09 & 17.33 & 3.51 & 243.48 \\
\hline 6.0 & 2.23 & 813 & 4.64 & 17.96 & 3.70 & 219.71 \\
\hline \hline
\end{tabular}

Tabla 6. Resumen ensayo Marshall para mezcla asfáltica MDC-2 con CA 60-70 Table 6. Marshall Test summary to asphalt mixture MDC-2 with AC 60-70

\begin{tabular}{|c|c|c|c|c|c|c||}
\hline $\begin{array}{c}\text { CA } \\
\text { AC } \\
{[\%]}\end{array}$ & $\begin{array}{c}\text { Peso unitario } \\
\text { Unit weight } \\
{\left[\mathrm{g} / \mathrm{cm}^{3}\right]}\end{array}$ & $\begin{array}{c}\text { Estabilidad } \\
\text { Stability } \\
(\mathrm{E})[\mathrm{kg}]\end{array}$ & $\begin{array}{c}\text { Vacíos en la mezcla } \\
\text { Vacuum M ixture } \\
{[\%]}\end{array}$ & $\begin{array}{c}\text { Vacíos en agregados } \\
\text { Vacuum on aggregate } \\
{[\%]}\end{array}$ & $\begin{array}{c}\text { Flujo/Fluid } \\
(\mathrm{F}) \\
{[\mathrm{mm}]}\end{array}$ & $\begin{array}{c}\text { Relación } \\
\text { Relation } \\
\text { E/F }[\mathrm{kg} / \mathrm{mm}]\end{array}$ \\
\hline 5.0 & 2.24 & 1189 & 5.83 & 16.95 & 3.40 & 349.86 \\
\hline 5.5 & 2.25 & 1323 & 4.47 & 16.80 & 3.60 & 367.84 \\
\hline 6.0 & 2.25 & 1393 & 3.48 & 16.96 & 3.69 & 377.18 \\
\hline 6.5 & 2.26 & 1229 & 3.02 & 17.60 & 3.92 & 313.38 \\
\hline
\end{tabular}

Tabla 7. Resumen ensayo Marshall para mezcla asfáltica M DC-2 con CAM 20-40 Table 7. Marshall Test summary to asphalt mixture MDC-2 with ACM 20-40

\begin{tabular}{|c|c|c|c|c|c|c||}
\hline $\begin{array}{c}\mathrm{CA} \\
\mathrm{AC} \\
{[\%]}\end{array}$ & $\begin{array}{c}\text { Peso unitario } \\
\text { Unit weight } \\
{\left[\mathrm{g} / \mathrm{cm}^{3}\right]}\end{array}$ & $\begin{array}{c}\text { Estabilidad } \\
\text { Stability } \\
(\mathrm{E})[\mathrm{kg}]\end{array}$ & $\begin{array}{c}\text { Vacíos en la mezcla } \\
\text { Vacuum M ixture } \\
{[\%]}\end{array}$ & $\begin{array}{c}\text { Vacíos en agregados } \\
\text { Vacuum on aggregate } \\
{[\%]}\end{array}$ & $\begin{array}{c}\text { Flujo/Fluid } \\
(\mathrm{F}) \\
{[\mathrm{mm}]}\end{array}$ & $\begin{array}{c}\text { Relación } \\
\text { Relation } \\
\text { E/F }[\mathrm{kg} / \mathrm{mm}]\end{array}$ \\
\hline 5.0 & 2.28 & 1398 & 4.28 & 15.51 & 3.67 & 380.93 \\
\hline 5.5 & 2.30 & 1492 & 2.53 & 15.01 & 3.74 & 398.93 \\
\hline 6.0 & 2.32 & 1507 & 0.92 & 14.67 & 4.00 & 376.75 \\
\hline 6.5 & 2.29 & 1431 & 1.70 & 16.38 & 4.38 & 326.71 \\
\hline
\end{tabular}




\section{Evolución de los parámetros de estabilidad y rigidez $\mathrm{M}$ arshall en el tiempo}

En la Figura la se observa la evolución en el tiempo ( $t$ en meses) de los parámetros de estabilidad del ensayo Marshall y en la figura $1 \mathrm{~b}$ la relación entre la estabilidad $(E)$ y el flujo $(F)$ (llamada por algunos investigadores como rigidez Marshall). Esta relación físicamente puede ser entendida como una resistencia mecánica evaluada en el estado de falla de las mezclas, bajo carga monotónica en un ensayo de tracción indirecta. Se observa para las mezclas fabricadas con CA 80-100, CA 60-70 y CAM 20-40 un incremento en la estabilidad con el tiempo de exposición al medio ambiente (ver Figura 1a). Las mezclas elaboradas con CA 60-70 en $t=17$ meses incrementa su estabilidad en un $81.6 \%$ su valor comparado con el alcanzado en $t=0$, aquellas fabricadas con CA 80-100 en $t=18$ meses incrementan en un $69.5 \%$ y las fabricadas con CAM 20-40 incrementan en $t=8$ meses en un $17.4 \%$.

\section{Time evolution of the Marshall stiffness and stability parameters}

Figure 1 shows the time evolution ( $t$ in months) of the Marshal stability parameters while Figure $1 \mathrm{~b}$ the relationship between stability ( $E$ in Spanish) and fluid $(F)$ (this relation is called by some researchers $\mathrm{M}$ arshall stiffness). This relation, in terms of physic may be understood as mechanical strength evaluated in the failure mixture state, under monotonic loading in an indirect tensile test. We can see in Figure $1 \mathrm{a}$ an increase of the stability along the exposure time for mixtures made with AC 80-100, AC 60-70 and ACM 20-40. The mixtures made with $A C$ 60-70 with time $t=17$ months, increase its stability in an $81.6 \%$ comparing values reached on $t=0$, those made with AC 80-100 increase to a $69,5 \%$ in $t=18$ months and those with ACM 20-40 increase a $17.4 \%$. $\mathrm{t}=8$ months

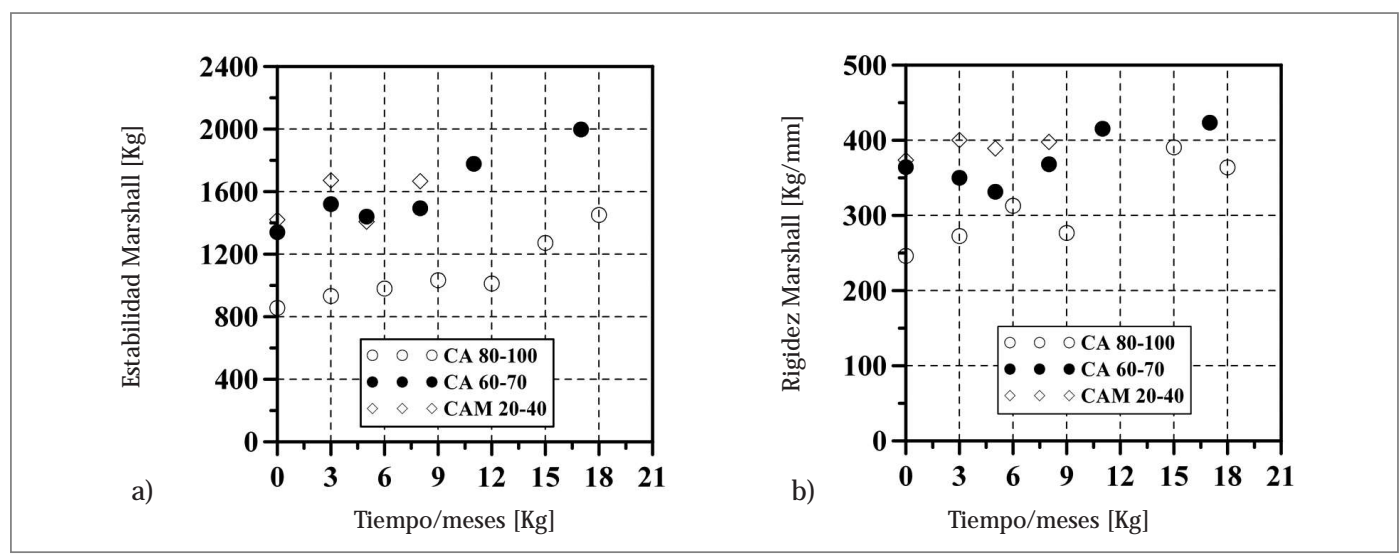

Figura 1. Evolución de a) estabilidad M arshall y b) relación E/F (rigidez M arshall) con el tiempo de envejecimiento, para mezclas fabricadas con CA 80-100, CA 60-70 y CAM 20-40

Figure 1. Evolution of a) M arshall Stability and b) Relation E/F (M arshall Stiffness) with aging Time for mixtures made with AC 80-100, AC 60-70 and ACM 20-40

En la Figura 1b se observa que la resistencia mecánica de las mezclas fabricadas con CA 60-70 tiende a aumentar con el tiempo de exposición, y en $t=17$ meses incrementa un $41.6 \%$ con respeto al valor inicial alcanzado en $t=0$. Para el caso de las mezclas con CA 80-100 y CAM 20-40 el incremento en $t=18$ meses y $t=8$ meses es de aproximadamente $53 \%$ y $14.9 \%$ respectivamente en comparación con el valor obtenido en $t=0$. Adicionalmente se observa un decaimiento de los valores de la relación $E / F$ en algunos periodos de tiempo lo cual puede ser explicado por dos fenómenos que están ocurriendo en las mezclas simultáneamente:
Figure $1 \mathrm{~b}$ shows that the AC 60-70 mechanical srtength presents a tendency to increase upon time exposure, and in $t=17$ months this raise a $41,6 \%$ in comparison with the initial value at $t=0$. In case of mixtures with AC 80-100 and ACM 20-40 the increase in $t=18$ months and $t=8$ months is approximately $53 \%$ and $14.9 \%$, respectively comparing it with the value shown in $t=0$. Additionally, a decay of the values related to $E / F$ in some periods can be observed, and this can be explained by two phenomena that happen simultaneously in the mixtures: 
- Envejecimiento por oxidación que experimenta el cemento asfáltico, y por lo tanto las mezclas, cuando es solicitado a diferentes gradientes de temperatura y exposición a radiación ultra-violeta (UV).

- El agua en las mezclas produce pérdida de adherencia entre el agregado pétreo y el asfalto, lo cual genera un aumento del flujo y disminución de la relación estabilidad - flujo.

\section{Evolución del módulo resiliente y la resistencia a la deformación permanente}

En la Figura 2 se observa la evolución del módulo resiliente de las mezclas asfálticas con el tiempo. Se observa para las mezclas fabricadas con CA 80-100, CA 60-70 y CAM 20-40 (Figuras $2 a$, b y c respectivamente) un incremento típico del módulo cuando se aumenta la frecuencia de carga y disminuye la temperatura del ensayo. Para el caso de las mezclas fabricadas con CA 80-100, el módulo tiende a aumentar con el tiempo de exposición al ambiente (ver figura 2a) y por lo tanto la resistencia a la deformación permanente aumenta (Figura 3). Este fenómeno puede ser debido principalmente al envejecimiento por oxidación que experimenta el cemento asfáltico por efectos de temperatura y exposición a radiación ultra-violeta (UV).

Las mezclas fabricadas con CA 60-70 y CAM 20-40 experimentan un comportamiento diferente en comparación con aquellas fabricadas con CA 80-100 (ver Figuras 2b-c y 3). En los primeros meses de exposición, las mezclas disminuyen su módulo aumentando las deformaciones permanentes, luego dicho módulo se estabiliza y por último la tendencia es a aumentar (especialmente cuando el ensayo se realiza con temperaturas entre 20 y $30^{\circ} \mathrm{C}$ ) tendiendo a disminuir los valores de deformación. El decaimiento en el módulo es debido tal vez a la generación de microfisuras que se producen a bajas temperaturas cuando las mezclas son rígidas (comportamiento frágil), luego estas microfisuras se estabilizan y el aumento en rigidez se debe a un fenómeno de envejecimiento y oxidación del asfalto similar al que ocurre en las mezclas con CA 80-100.
- Aging by oxidation experienced by asphalt cement and consequently by the asphalt mixtures, when it is required in different temperatures and exposure to ultra-violet radiation (UV).

- Water in mixtures produces loss of bonding between aggregate and asphalt cement which gives place an increase in fluid and diminution of the fluid-stability relation.

\section{Evolution of resilient modulus and resistance to permanent deformation}

The time evolution of the asphalt mixture modulus is observed on Figure 2. It should be noticed that for mixtures made with CA 80-100, CA 60-70 and CAM 20-40 (Figures $2 a, b$ and c, respectively) there is a typical modulus increase as the loading frequency raises and test temperature decreases. In the case of mixtures made with CA 80-100, the modulus tendency is to increase with the environmental exposure (Figure 3). This phenomenon may be explained mainly by the aging by oxidation that asphalt experiments due to temperature and ultraviolet ray exposure (UV).

The mixtures made with $A C$ 60-70 and ACM 20-40 experienced a different behaviour in comparison with those with AC 80-100 (see Figures 2b-c and 3). During the first months of exposure, the resilient modulus decreased which increased permanent deformations. After that, the modulus stabilized; and finally, the tendency is to increase (specially when the test is made at 20 and $30^{\circ} \mathrm{C}$ ), wich helps to decrease the deformation values. The modulus decrease may be explained based on the microcracks produced at low temperatures when the mixture is rigid (brittle behaviour); then, these microcracks stabilize and stiffness increases because of a similar phenomenon of aging and oxidation that happens in mixtures with AC 80-100. 


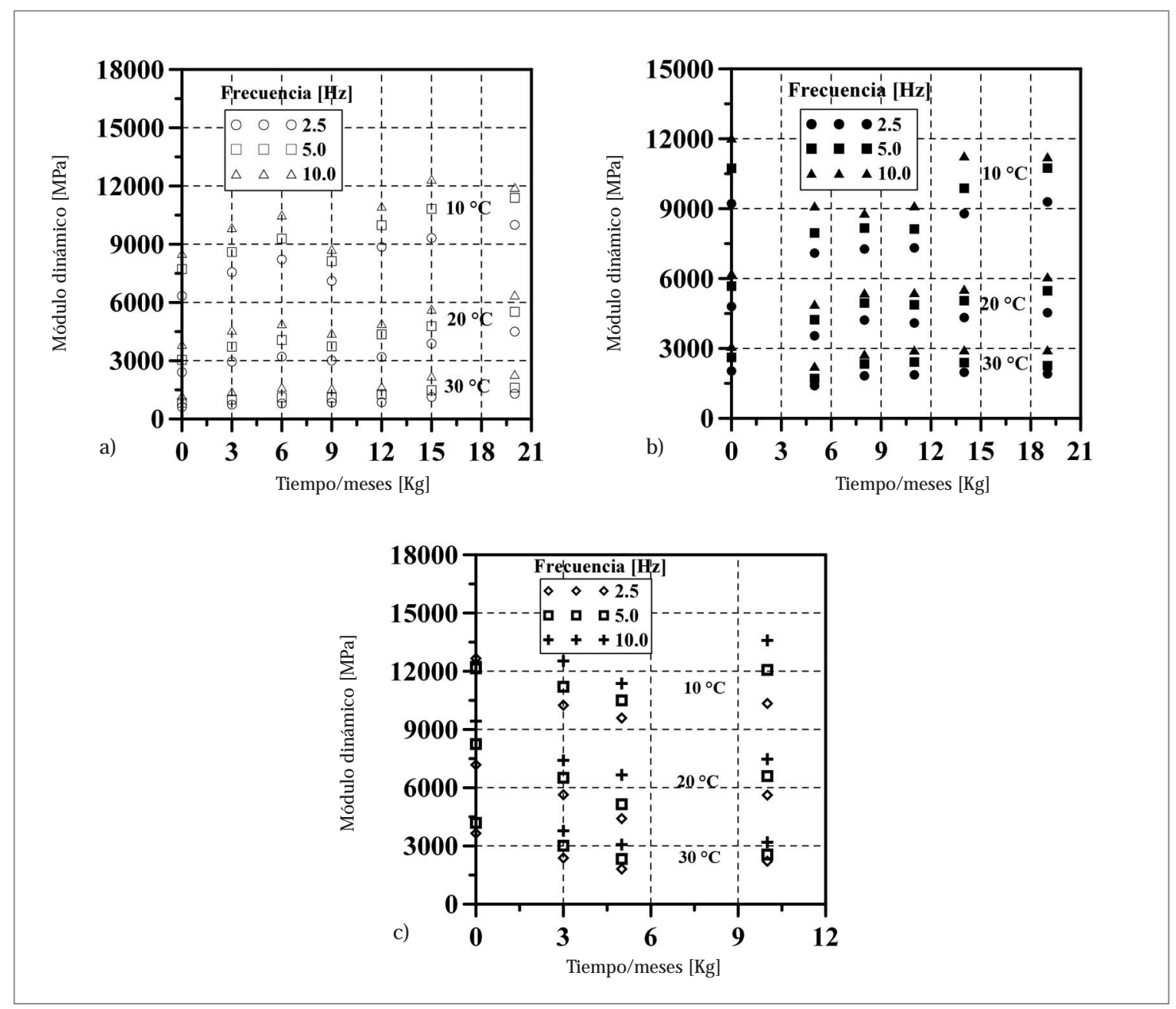

Figura 2. Evolución del módulo resiliente con el tiempo de envejecimiento para mezclas fabricadas con a) CA $80-100$, b) CA $60-70$ y c) CAM 20-40

Figure 2. Resilient modulus evolution with aging time for mixtures made with a) AC 80-100, b) AC 60-70 and C) ACM 20-40

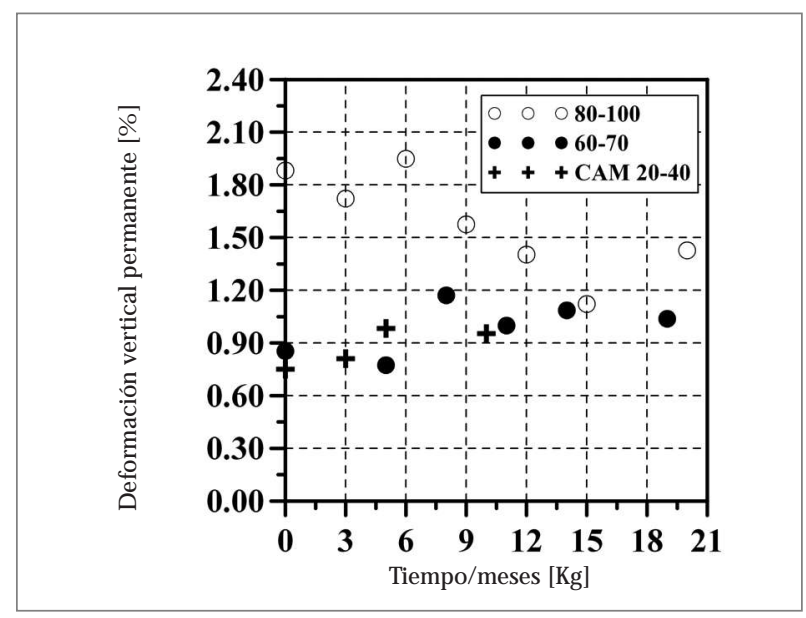

Figura 3. Evolución de la deformación vertical permanente con el tiempo de envejecimiento para mezclas fabricadas con CA 80-100, CA 60-70 y CAM 20-40

Figure 3. Evolution of permanent vertical deformation with aging time for mixtures made with AC 80-100, AC 60-70 and MAC 20-40 
El efecto del agua no es claro en los resultados observados ya que la humedad en las mezclas produce pérdida de adherencia entre el agregado pétreo y el asfalto, generando una posible disminución del módulo y de la resistencia mecánica. Investigaciones adicionales en esta área deben ser realizadas.

En la figura 4 se presenta la relación $\left(E_{D} / E_{D o}\right)$ entre el módulo resiliente que se obtiene luego de someter las briquetas al ambiente durante distintos periodos de tiempo $(E D)$ y el módulo resiliente inicial de las mezclas en un tiempo $t=0$ meses $\left(E_{D o}\right)$. Para el caso de las mezclas con CA 80-100, el módulo alcanzado en $t=20$ meses aumenta entre un 40 y $110 \%$ (dependiendo de la temperatura y frecuencia del ensayo) con respecto al inicial. Las mezclas con CA 60-70 experimentan una disminución máxima en su módulo de $35 \%$ en los primeros cinco meses de exposición para luego aumentar y experimentar en $t=19$ meses, módulos que tienden a alcanzar el valor inicial. U n comportamiento similar al que experimentan las mezclas fabricadas con CA 60-70 se observa en las mezclas con CAM 20-40. Ecuaciones empíricas regresionales que pueden reproducir la evolución de los módulos con el tiempo son formuladas en (1) para el caso de mezclas con CA 80-100 y (2) para CA 60-70 y CAM 20-40. U na simulación de los resultados expuestos empleando las ecuaciones (1) y (2) se presenta en la Figura 4 a y b. En la ecuación (1), $a=1.0$ y $b=0.153$; en la ecuación (2), $a=0.524, \mathrm{~b}=0.102, c=0.476$ y $a=0.713$, $b=0.021$ y $c=0.289$ para las simulaciones de las mezclas con CA 60-70 y CAM 20-40 respectivamente. El coeficiente de correlación de las ecuaciones (1) y (2) es $r^{2}=0.77$.
There is no clear effect of water on the results because the humidity produces bonding loss between aggregate and asphalt cement, giving place to a possible module and mechanical srtenght decrease. There should be additional research in this area.

Figure 4 shows the relation $\left(E_{D} / E_{D o}\right)$ between the resilient mixture modulus obtained after environmental exposure in different periods of time $(E D)$ and the initial resilient mixture modulus at time of $t=0$ months $\left(\mathrm{E}_{D o}\right)$. The $t=20$ modulus reaches, in the case of mixtures with AC 80-100, increases a 40 and $110 \%$ (depending on test and frequency temperatures) compared with the initial value. M ixtures with AC 60-70 experienced a maximum modulus decrease of $35 \%$ during the first five months of exposure and then an increase that nearly reached the initial modulus value at $t=19$ months. The mixtures made with ACM 60-70 showed a similar behaviour to those made with AC 20-40. It can be observed in Equation (1), for the case of mixtures made with $A C 80-100$, and in Equation (2), for mixtures made with AC 60-70 y ACM 20-40, empirical equations that may be producing the evolution in modulus with time. There is a simulation of the results shown using equations ( 1 ) and (2) on Figure $4 \mathrm{a}$ and $\mathrm{b}$. In the equation (1), $a=1.0$ and $b=0.153$; in the equation (2), $a=0.524, b=0.102, c=0.476$ and $a=0.713$, $b=0.021$ and $c=0.289$ for simulations of mixtures with CA $60-70$ and CAM 20-40, respectively. The correlation coefficient of equations is $r^{2}=0.77$.

$$
\begin{gathered}
\frac{E_{D}}{E_{D o}}=a+b \sqrt{t} \\
\frac{E_{D}}{E_{D o}}=a+b \sqrt{t}+c \exp ^{-t}
\end{gathered}
$$

Como se ha demostrado en otras investigaciones (p.e., Afanasieva y Àlvarez, 2004; Vargas et al., 2008), el envejecimiento oxidativo ejerce una gran influencia en las propiedades viscoelásticas del cemento asfáltico reflejado en el incremento del módulo elástico G*, resultado del aumento de las interacciones moleculares del asfalto como: fuerzas dispersivas, puentes de hidrógeno e interacciones polares, especialmente de los asfaltenos,
The aging by oxidating have an important influence on the asphalt cement viscoelastic properties, as demonstrated by other researches (Afanasieva y Àlvarez, 2004; Vargas et al., 2008). This influence is shown in the $\mathrm{G} *$ elastic modulus increase, produced by the increase in the asphalt molecular interaction: dispersed forces, hydrogen bridges and polar interactions, especially in asphaltenes, which give a better asphalt structuration or increase the 
que en conjunto resultan en una mayor estructuración del asfalto o un incremento en la conectividad de las moléculas del material. Las temperaturas bajas $\left(<40^{\circ} \mathrm{C}\right)$ a su vez favorecen el incremento de la rigidización del asfalto que junto con la influencia de los diferentes factores climáticos de la ciudad de Bogotá D.C. explican el comportamiento observado en las mezclas asfálticas usadas en este estudio. El comportamiento de la relación $\left(E_{D} / E_{D o}\right)$ observado en las mezclas con CA 60-70 y CAM 20-40 puede deberse a que éstos materiales deben presentar un menor contenido de fracciones livianas susceptibles de ser envejecidas contrario al CA 80-100. Es decir, la tendencia al cambio de su composición química con el tiempo de envejecimiento puede ser menor y esto se ve reflejado en las propiedades mecánicas de la mezcla asfáltica diseñada con este asfalto. Este hecho necesita ser estudiado a partir de la determinación del cambio de la composición química del CA bajo las condiciones climáticas de Bogotá D.C. material molecule connectivity. Low temperatures $\left(<40^{\circ} \mathrm{C}\right)$, facilitate asphalt rigidization which combined with the Bogota's different weather conditions, explain the observed behaviour of the asphalt mixtures used in this research. The behaviour of the $E_{D} / E_{D o}$ relationship observed in AC 60-70 and ACM 20-40 mixtures may be explained based on the fact that these materials should present a lower content of lightweight fractions that are susceptible of aging, as opposed to AC 80-100. That is, its chemical composition tendency to change with aging may be lower which shown by the mechanical properties of the asphalt mixture designed with this asphalt. This fact needs an analysis beginning with the AC chemical composition under the Bogotá D.C's weather conditions.

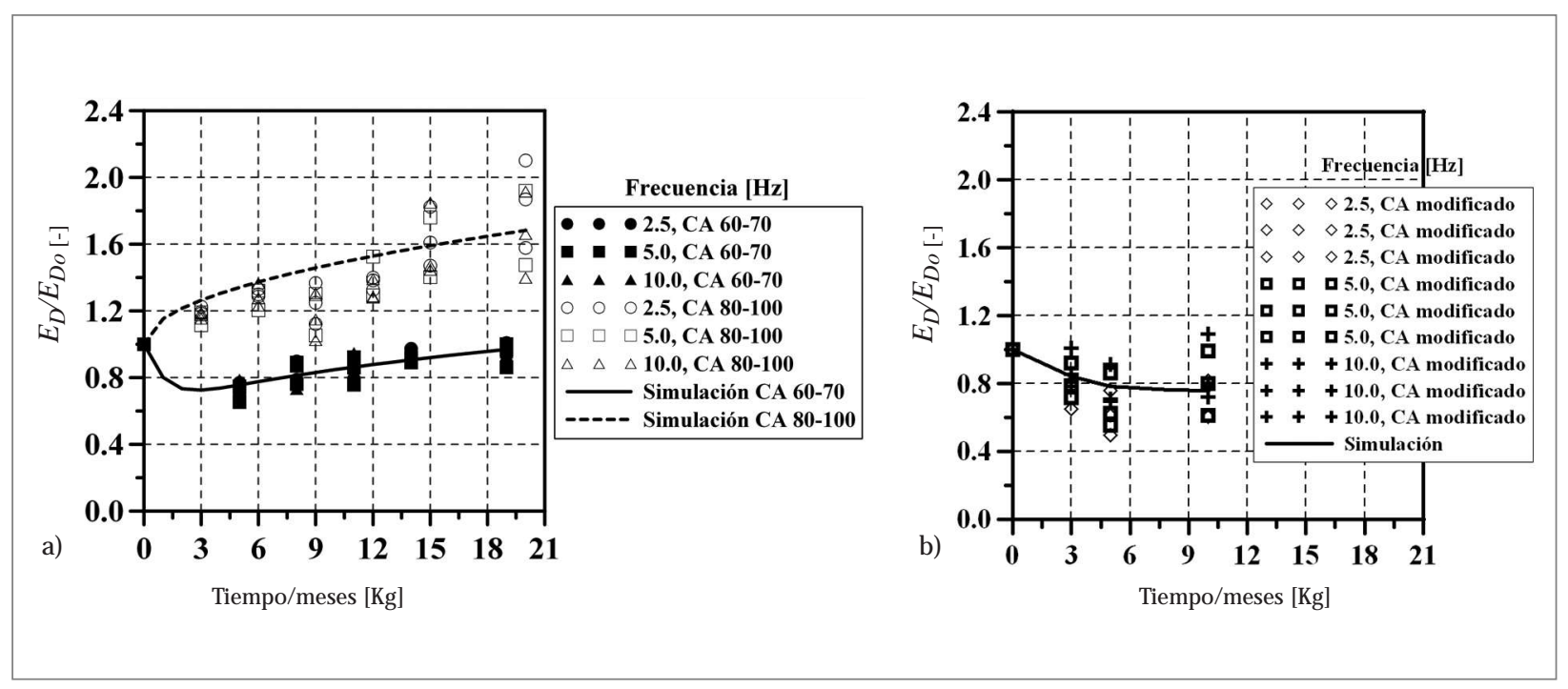

Figura 4. Evolución de la relación entre el módulo resiliente y el módulo inicial con el tiempo de envejecimiento para mezclas fabricadas con a) CA 80-100 y CA 60-70, b) CAM 20-40 Figure 4. Evolution of the relation of between resilient and initial modulus with aging time for mixtures made with a) AC 80-100 and AC 60-70, b) ACM 20-40 


\section{Conclusiones}

En el artículo se presenta la primera fase de un proyecto de investigación que tiene como objetivo principal evaluar el cambio en las propiedades mecánicas que experimenta una mezcla asfáltica densa en caliente cuando se exponen a condiciones reales del medio ambiente de la ciudad de Bogotá D.C. En esta primera fase se evalúa la evolución cada tres meses, durante 20 meses, del módulo resiliente, la resistencia a la deformación permanente y los parámetros del ensayo Marshall de mezclas tipo MDC-2 fabricadas con CA 80100 y CA $60-70$.

Las mezclas fabricadas con CAM 20-40 el tiempo de evaluación ha sido de 10 meses. De manera general se observa que las mezclas fabricadas con los tres tipos de CA utilizados incrementan su resistencia mecánica bajo carga monotónica (evaluado a través de la estabilidad y la relación $E / F$ del ensayo Marshall) con el tiempo de exposición al ambiente.

Para el caso de las mezclas fabricadas con CA 80-100 el módulo resiliente y la resistencia a la deformación permanente tiende a aumentar en el tiempo debido principalmente a fenómenos de envejecimiento por oxidación del asfalto.

Mezclas elaboradas con CA 60-70 y CAM 2040 , en los primeros cinco meses de exposición disminuyen su módulo resiliente aumentando las deformaciones permanentes (debido tal vez a microfisuración por bajas temperaturas), luego se estabiliza entre el quinto y octavo mes de exposición y por último la tendencia es a aumentar debido al endurecimiento que experimenta el ligante asfáltico por fenómenos de oxidación (envejecimiento).

Las fases futuras del proyecto deben medir y evaluar la evolución de parámetros mecánicos como resistencia a fatiga de las mezclas asfálticas, así como el cambio en la composición química que experimentan los cementos asfálticos utilizados para la fabricación de las mezclas.

\section{Conclusions}

This article represents the first stage of a research project, which objective is to evaluate the mechanic property changes that a hot asphalt mixture experiences when exposed to real environmental conditions at Bogotá D.C city. In this first stage there were evaluations every three months, during the first 20 months, checking, resilient modulus, resistance to permanent deformation and $\mathrm{M}$ arshall test parameters on mixtures classified as MDC-2 made with AC 80-100 and AC 60-70.

The evaluation for mixtures made with $\mathrm{ACM}$ 20-40 was 10 months. It can be observed that mixtures made with the three AC type used, increase its mechanical strength under monotonic loading (evaluated through the relation $E / F$ of Marshall test) with the environment exposure time.

In the case of mixtures made with 80-100 resilient modulus and resistance to permanent deformation tendency is to increase with time mainly because of asphalt oxidation phenomenon.

Mixtures made with AC 60-70 and ACM 20-40, decreased their resilient modulus during the first months of exposure increasing permanent deformation (this is may be due to low temperature microcracking); after that, an stabilization in the resilient modulus is shown within fifth and eight months of exposure; and finally, a tendency to increased resilient modulus is shown due to the toughness experienced by the asphalt oxidation phenomenon (aging).

The future project steps should measure and evaluate the evolution of mechanical parameters such the resistance of asphalt mixture to fatigue, and the chemical composition of the asphalt cements used in the mixtures experiment.

\section{Referencias / References}

Afanasieva N. y Álvarez M. (2004), Estudio del envejecimiento de los asfaltos bajo la acción de algunos factores climáticos. U niversidad Industrial de Santander, Bucaramanga, Colombia.

Airey G. D. (2003), State of the art report on ageing test methods for bituminous pavement materials. International Journal of Pavement Engineering 4, No 3, 165-176.

Bishara S. W., Robertson R. E. y Mahoney D. (2000), Rapid oxidative aging of binder using microwave energy - An improved method. Proc. 2nd Eurasphalt and Eurobitume Congress, Session 2: Development in Bituminous Products and Techniques, (Barcelona), 27-36. Bocci M. y Cerni G. (2000), The ultraviolet radiation in short-and long-term aging of bitumen. Proc. 2nd Eurasphalt and Eurobitume Congress, Session 1: Performance Testing and Specifications for Binder and Mixtures (Barcelona) 49-58. Brown S. F. y Scholz T. V. (2000), Development of laboratory protocols for the ageing of asphalt mixtures. Proc. 2nd Eurasphalt and 
Eurobitume Congress, Session 1: Performance Testing and Specifications for Binder and Mixtures, (Barcelona) 83-90. CEN - Committee European of N ormalization. (2000), Bituminous mixture test methods for hot mix asphalts - Part25: Cyclic compression tests.

Choquet F. S., y Verhasselt A. F. (1992), N atural and accelerated ageing of bitumens - effects on the asphaltenes. Proc. of an International Symposium on Asphaltene particles in Fossil Fuel Exploration, Recovery, Refining and Production Processes, Ed. MK Sharna and TF Yen 1994, Plenum Press, N ew York, 13-29.

INVIAS - Instituto Nacional de Vías. (2007), Especificaciones generales de Construcción de carreteras. Bogotá D.C. INVIAS - Instituto Nacional de Vías. (2007a), Normas de Ensayos de Materiales para Carreteras. V. I y II. Bogotá D.C. Jemison H. B., Davison R. R., Glover C. J., y Bullin J. A. (1991), Evaluation of Standard O ven Tests for hot-mix plant aging. Transportation Research Record, 1323, Transportation Research Board, Washington D.C., 77-84.

Kemp G. R., y Predoehl N. H. (1981), A comparison of field and laboratory environments of asphalt durability. Proc. Assn. Asphalt Paving Technol., 50, 492-537.

Khalid H. A. (2002), A new approach for the accelerated ageing of porous asphalt mixtures. Proceedings of the Institution of Civil Engineers, Transport 153, Issue 3, 171-181.

Khalid H. A. y Walsh C. M. (2000), Relating mix and binder fundamental properties of aged porous asphalt materials. Proc. 2nd Eurasphalt and Eurobitume Congress, Session 1: Performance Testing and Specifications for Binder and Mixtures (Barcelona), $398-405$.

Kim O-K., Bell C. A., Wilson J. E. y Boyle G. (1987), Development of laboratory oxidative aging procedures for asphalt cements and asphalt mixtures. Transportation Research Record, 1115, Transportation Research Board, Washington D.C., $101-112$.

Kuppens E. A. M., Sanches F., N ardelli L., y Jongmans E. C. (1997), Bitumen-ageing tests for predicting durability of porous asphalt. In: Di Benedetto, H., Francken, L., Proc. Fifth International RILEM Symposium, Mechanical Tests for Bituminous Materials, 71-77.

Migliori F. y Corté J. F. (1998), Comparative study of RTFOT and PAV aging simulation laboratory tests. Transportation Research Record, 1638, Transportation Research Board, Washington D.C., 56-63.

M ontepara A. (1999), A theoretical-interpretative model of the relationship between UV-radiation ageing of bitumen and viscosity variation. Proc. of Eurobitume Workshop, paper N o. 075, Luxemburg.

Montepara A. y Giuliani F. (2000), Comparison between ageing simulation tests of road bitumen. Proc. 2nd Eurasphalt and Eurobitume Congress, Session 1: Performance Testing and Specifications for Binder and Mixtures, (Barcelona), 518-523.

Shiau J-M., Tia M., Ruth B. E. y Page G. C. (1991), Characterization of age-hardening potential of asphalt by using Corbett-Swarbrick Asphalt Fractionation Test. Transportation Research Record, 1323, Transportation Research Board, Washington D.C., 53-60.

Said S. F. (2005), Aging effect on mechanical characteristics of bituminous mixtures. Transportation Research Record: Journal of the Transportation Research Board, 1901, Washington D.C., 1-9.

Shen J., Amirkhanian S. y Tang B. (2006), Influence of accelerated aging test temperature on the properties of binders. International Journal of Pavement Engineering, 7, Issue 3, 191-198.

Vargas X. A., Afanasjeva N., Álvarez M., Marchal P. H. y Choplin L. (2008), Asphalt rheology evolution through thermo-oxidation (aging) in a rheo-reactor. FUEL, 87, issues 13-14, 3018-3023.

Verhasselt A. F. (1997), Field Ageing of Bituminous Binders: Simulation and Kinetic approach. In: Di Benedetto, H., Francken, L., Proc. Fifth International RILEM Symposium, Mechanical Tests for Bituminous Materials, 121-128.

Welborn J. Y. (1984), Physical properties as related to asphalt durability: State of the art. Transportation Research Record, 999, Transportation Research Board, Washington D.C., 31-36. 